\title{
An interactive gaming package for teaching and research in interdependent choice
}

\author{
PAUL E. SCHAFFNER \\ Bowdoin College, Brunswick, Maine
}

\begin{abstract}
A pair of FORTRAN programs allows two individuals or groups to play prisoner's dilemma and other two-party mixed-motive games on physically isolated terminals of a time-sharing computer. The programs provide a flexible tool for teaching and research through a series of programmable options for structuring and enhancing the playing environment of the game. Four participatory roles for students provide an opportunity for integrated exposure to the broader context of empirical research design and execution.
\end{abstract}

Two FORTRAN IV programs and supporting materials allow two players using an interactive time-sharing computer system to play any two-party mixed-motive game under a variety of conditions and rules. The gameplaying program is available to any user without supervision, as is limited access to the package's game-structure modification (setup) program. Full access to the setup program is available by providing an unrequested password. Users who do so have greater flexibility in designing the characteristics of subsequent game play; they can also elect to have each party independently play against a program of preselected moves instead of against the other player. Extensive research flexibility is thus available for studying interdependent choice over time.

These materials have been developed since 1980 for use in classroom demonstrations, continuing laboratory exercises, and independent research projects. They are suitable for use by either individual players or small groups. The package's hierarchical control format for structuring game play allows students to move through a sequence of four levels of direct involvement in experimental research.

\section{PACKAGE DESIGN AND OPERATION}

The package is designed to allow two naive users to access and play some game at any time, without supervision and without necessarily discovering the availability of most of the package's structuring options. When the package is used in this manner, one player logs onto the computer system, assigns a second terminal to his or her job, and runs the compiled game-playing program available on line. The second terminal is slave to the first, but

This material is based upon work supported by the National Science Foundation under Grant SER-8001451. Christopher Loughlin provided extensive programming assistance in the development of the programs described here. The author's mailing address is: Psychology Department, Bowdoin College, Brunswick, Maine 04011 . this relationship is not noticeable once play is underway. Two levels of greater flexibility are available through the setup program. In its simpler mode of operation, the setup program informs the logged-in player of the availability of some options for structuring the game, such as the number of rounds and choice among payoff matrices. Once appropriate choices are made, the setup program calls the playing program to begin a game.

The greatest power of the package is available to a researcher or instructor who enters " 2500 " in response to the setup program's request for the current time of day. He or she may then select any combination of conditions from a menu of options, without necessarily informing the players of the availability of these or alternative game configurations. Users subsequently playing the game will encounter whatever features have been selected most recently. The researcher may choose to have most option categories be made accessible to anyone running the setup program. For each option category made accessible in this way, instructions for choosing particular game features are then presented to anyone who runs the setup program. Options are selected from a menu format with review, reset, and default capabilities. Any run of the playing program uses whater game configuration has been entered into the setup data file most recently.

Operation of the playing program is straightforward. The two players or teams, preferably at physically isolated terminals, are shown game instructions and a payoff matrix, and are usually informed of the number of rounds in the game and of capabilities they might have for communicating with, rewarding, or penalizing their playing partners. When the game begins, each player simply enters " $A$ " or " $B$ " choices. After both players have chosen on each round, results are displayed on both terminals. At the conclusion of the game, a cumulative tally is displayed and the logged-in player is prompted with the option of a new game.

Record keeping is automatic and unannounced. The playing program copies an existing output. file at the 
beginning of each game and then adds to it the current game's record of player choices, points earned and assigned, and messages. The updated file is output on disk at the end of each game. This arrangement assures that if either player interrupts the game, only the most recent game record will be lost.

\section{GAMESTRUCTURING OPTIONS}

The "matrix option" allows the user to select any of six prominent payoff matrices from a menu that includes prisoner's dilemma, chicken, Castor and Pollux, hero, leader, or battle of the sexes. The program allows one to enter new values to create any matrix; thus, any of the 78 distinct two-party two-choice matrices identified by Rapoport and Guyer (1966) as constituting the universe of such decision settings can be used in this package. The option of choosing a matrix is typically made available to all players running the setup program.

The "reward/penalty option" makes reward and/or penalty points available to both players or to only one player to assign to the other after each round of play. Players decide on reward or penalty point values on each round within adjustable limits. Rewarding and penalizing are independent functions; they can be added individually or together. If included, the opportunity to reward or penalize the other player can be scheduled either to precede or to follow feedback to players on each prior round's outcome.

The "communication option" allows each player, or only one, to send messages to the other player between rounds. The game limit for messages is adjustable. Message content is unrestricted but is limited to one line per message. When both players are able to send messages, the playing program alternates opportunities to transmit.

The "cumulative feedback option" determines how often, if ever, cumulative feedback is presented. Currentround feedback and a game-end tally are always presented.

The "data file option" allows either player to store a simple data file, containing the record of choices and payoffs for the game, on the logged-in user area. It is independent of the master record file, which is always maintaine in another user area.

The "reversal option" allows one to indicate specific rounds on which one player's choice (A or B) will be reversed. Reversals may be scheduled for either player or both; they are neither announced nor explained. This allows the researcher to study responses by either player to an unintended choice.

The "programmed play option" unobtrusively substitutes a preselected pattern of computer-generated responses for each player's ostensive partner. Play patterns are chosen from a menu that includes same-choice, opposite-choice, echoed same-choice (tit-for-tat), A-B alternation, all-A or all-B, and other patterns. Randomly scheduled random-choice play can be superimposed on any programmed play pattern, with adjustable frequen. cies and adjustable $\mathrm{A} / \mathrm{B}$ probabilities. When programmed play is elected, the same program of computer choices is followed for each of the two simultaneous but independent games being played. For obvious reasons, programmed play and communication cannot both be selected for the same game.

The "unknown endpoint option" suppresses initial game instructions informing players of the number of rounds to be played. This eliminates the strategic complications introduced by knowledge of which round ends a game; end-game strategy varies both normatively and empirically from play during earlier rounds.

\section{APPLICATIONS, FINDINGS, AND EVALUATION}

The package has been used in several educational contexts. These include introductory psychology demonstrations, laboratory exercises in social and political psychology and behavioral science modeling, and individual and class independent research projects. Handouts that require students to record the progress of their games as they are played, and to interpret game outcomes after the fact, have led to some detailed and insightful reports.

Students learn most from the package by progressing through four levels of involvement in sequence: individual player, team member, research supervisor (overseeing the execution of a given research design), and research designer (creating new studies using various combinations of package options). To date, advanced undergraduates have investigated topics including singlestep transition probabilities, manipulative uses of communication, and the tendency to claim responsibility after the fact for programmed reversals of one's intended choices.

Although initial instructions to players emphasize the single goal of maximizing in dividual point values, novice play tends to shift quickly to highly competitive choices and to a standard of comparative, as opposed to absolute, success. This orientation is well-known in the research literature (Messick \& Thorngate, 1967). Adding weak extrinsic incentives based on absolute performance helpes to restore balance to the structurally conflicting motives inherent in the payoff matrices. Without them, students typically lock into mutually destructive B-B choice pattems interrupted only by occasional unreciprocated attempts to shift to cooperative choice. Lockingin is another common outcome in prisoner's dilemma research, especially among novice players (Rapoport \& Chammah, 1965).

There are three programming benefits of this package. First, experimental control and variation are greatly simplified. Initial and ongoing instructions, stimulus presentation, response collection, feedback, and data recording are standardized and automated. Second, communication among the players, when selected, is restricted to the single channel of typed messages, yet is without restrictions on content within that channel. Third, access to the package does not require supervi- 
sion, scheduling, or restriction to one pair of players at a time.

Using the computer for this package also has its drawbacks. Its time-sharing environment means that at least one person must know how to access the system and assign a second terminal to his or her job. This is not a problem when the package is being used in supervised research, but it can be a problem when players are expected to access and use the package without supervision. The use of terminal keyboards rather than simpler devices can lead to stray entries, several of which can abort play. Long delays sometimes occur at the beginning of a game, when novice users are unfamiliar with computer terminals or two-by-two choice matrices (or both). This can lead them to enter messages and plays prematurely. Such problems are efficiently addressed by including a brief practice game at the beginning of each session involving novices.

Student evaluations of courses in which the package has been used indicate positive but highly variable reactions to it. Combinations of laboratory experience, class discussions and postplay assignments calling for reflective interpretation help ensure that students generalize their experiences beyond the immediate context of an. abstract computer exercise. Future developments will include a restructuring of the automated data storage to facilitate Markov chain analysis of play under varying conditions (Van der Sanden, 1978), and possibly a more general package that can handle several players in $\mathrm{N}$-party gaming play (Hamburger, 1973).

The package is currently implemented on a DEC1091 computer, using the TOPS-10 operating system, including several file-accessing utilities. The setup and play programs compile in under $10 \mathrm{~K}$ of main memory each. Two sequential-access data files, containing the structuring information for a game and the cumulative record of play, are maintained by the two programs. Documented source code is available from the author.

\section{REFERENCES}

Hamburger, H. (1973). N-person prisoner's dilemma. Journal of Mathematical Sociology, 3, 27-48.

MEssick, D., \& Thorngate, W. (1967). Relative gain maximization in experimental games. Journal of Experimental Social Psychology, 3, 85-101.

Rapoport, A., \& Снамmaн, A. (1965). Prisoner's dilemma. Ann Arbor: University of Michigan Press.

RApopont, A., \& GuYen, M. J. (1966). A taxonomy of $2 \times 2$ games. General Systems, 11, 203-214.

VAN Der SANDen, A. L. (1978). Latent Markov chain analysis of a value conflict in prisoner's dilemma games. British Journal of Mathematical and Statistical Psychology, 31, 126-143. 